\title{
Recursion Theory in Set Theory
}

\author{
Theodore A. Slaman
}

\section{Introduction}

Our goal is to convince the reader that recursion theoretic knowledge and experience can be successfully applied to questions which are typically viewed as set theoretic. Of course, we are not the first to make this point. The detailed analysis of language, the absoluteness or nonabsoluteness of the evaluation of statements, and the interaction between lightface and relativized definability are thoroughly embedded in modern descriptive set theory. But it is not too late to contribute, and recursion theoretic additions are still welcome. We will cite some recent work by Slaman, Hjorth, and Harrington in which recursion theoretic thinking was applied to problems in classical descriptive set theory.

It is the parameter-free or lightface theory that seems closest to our recursion theoretic heart. Where another might see a continuous function, we see a function which is recursive relative to a real parameter. In the same way, we can see the Borel sets through the hyperarithmetic hierarchy and the co-analytic sets by means of well-founded recursive trees. We will make our way through most of the relevant mathematical terrain without invoking concepts which are not natively recursion theoretic. At the end, we will mention some problems which are similarly accessible.

We owe a debt to Sacks's (1990) text on higher recursion theory and to Kechris's (1995) text on descriptive set theory. These are valuable resources, and we recommend them to anyone who wishes to learn more about what we will discuss here. In the following, we will cite theorems from the nineteenth and early twentieth centuries without giving the original references; the motivated reader can find the history of descriptive set theory well documented in these texts.

\section{The Classical Theory}

Here is the framework. We speak exclusively about subsets of Baire space, ${ }^{\omega} \omega$, and refer to an $\omega$ sequence of natural numbers as a real number. A basic open set $B(\sigma)$ is determined by a finite sequence $\sigma$ from $\omega^{<\omega}: x \in B(\sigma)$ if and only if $\sigma$ is an initial segment of $x$. A function $f:{ }^{\omega} \omega \rightarrow{ }^{\omega} \omega$ is continuous if any finite initial segment of $f(x)$ is determined by a finite initial segment of $x$. If we think of this

Slaman wishes to thank L. Harrington, G. Hjorth, J. Steel, and W. H. Woodin for the information and advice that they provided during the preparation of this paper. Slaman was also partially supported by NSF Grant DMS-97-96121. 
correspondence between the argument and the domain as having been coded by a real number, then $f$ is recursive relative to that real. Conversely, if $f$ is is recursive relative to some real parameter, then $f$ is continuous.

Definition 2.1. 1. The Borel subsets of ${ }^{\omega} \omega$ are those sets which can be obtained from open sets by a countable iteration of countable union and complementation.

2. The analytic sets are the continuous images of the Borel sets.

The classical notions correspond to levels in the descriptive hierarchy of second order arithmetic.

Definition 2.2. $\quad$ 1. $A$ is a $\boldsymbol{\Sigma}_{1}^{1}$ set if and only if membership in $A$ is definable as follows.

$$
x \in A \Longleftrightarrow(\exists w)(\forall n) R(n, x \uparrow n, w \uparrow n, a \uparrow n)
$$

where $a$ is a fixed element of ${ }^{\omega} \omega, w$ ranges over ${ }^{\omega} \omega, n$ ranges over $\omega$, and $R$ is recursive.

2. $A$ is a $\boldsymbol{\Delta}_{1}^{1}$ set if and only if both $A$ and its complement are $\boldsymbol{\Sigma}_{1}^{1}$ sets.

Here is the connection. A set $C$ is closed if and only if there is an $a \in \omega^{\omega} \omega$ and there is a recursive predicate $R$, such that for all $x$,

$$
x \in A \Longleftrightarrow(\forall n) R(n, x \uparrow n, a \uparrow n) .
$$

By a classical fact, for every analytic set $A$, there is a closed set $C$ such that for all $x, x \in A$ if and only if there is a witness $w$ such that $(x, w) \in C$. Thus, a set is analytic if and only if it is $\boldsymbol{\Sigma}_{1}^{1}$.

Similarly, by a classical theorem of Suslin, the Borel sets are exactly those analytic sets whose complements are analytic. Consequently, $B$ is Borel if and only if it is $\boldsymbol{\Delta}_{1}^{1}$.

Definition 2.3. The projective sets are obtained from the Borel sets by closing under continuous images and complements.

Similarly to the above, the projective sets are those sets which can be defined in second order arithmetic using real parameters.

Initially, the projective sets were studied topologically. Much of the progress was limited to the Borel sets and the $\Sigma_{1}^{1}$ sets, for which a variety of regularity properties were established.

2.1. Perfect set theorems. Recall, a set $P$ is perfect if it is nonempty, closed, and has no isolated points. Equivalently, $P$ is perfect if and only if there is a (perfect) tree $T \subseteq \omega^{<\omega}$ such that every element of $T$ has incompatible extensions in $T$ and $P$ is $[T]$, the collection of infinite paths through $T$.

TheOREM 2.4. 1. (Cantor-Bendixson) Every uncountable closed subset of $\omega_{\omega}$ has a perfect subset.

2. (Alexandrov, Hausdorff) Every uncountable Borel subset of ${ }^{\omega} \omega$ has a perfect subset.

3. (Suslin) Every uncountable analytic subset of $\omega_{\omega} \omega$ has a perfect subset.

Suslin's Theorem follows directly from the representation of analytic sets given in 1. Suppose that $A$ is uncountable and is defined by

$$
x \in A \Longleftrightarrow(\exists w)(\forall n) R(n, x \uparrow n, w \uparrow n, a \uparrow n) .
$$


We build a tree $T$ of pairs $(\tau, \sigma) \in \omega^{<\omega} \times \omega^{<\omega}$ such that if $(\tau, \sigma) \in T$ then there are uncountably many $x$ extending $\tau$ for which there is a $w$ extending $\sigma$ such that $(\forall n) R(n, x\lceil n, w\lceil n, a\lceil n)$. We use the fact that $A$ is not countable to ensure that the projection of $T$ onto the first coordinates of its elements is a perfect tree $T_{1}$. Each path $x$ through $T_{1}$ is an element of $A$, as it is associated with a witness $w$ to that fact in $T$.

2.2. Representations of Borel sets. A diagonal argument shows that there is no universal Borel set. However, in a different sense, the Borel sets are closer to having a universal element than one might have thought.

Theorem 2.5 (Luzin-Suslin). For every Borel set B, there is a closed set $C$ and a continuous function $f$ which maps $C$ bijectively to $B$.

Proof. Whether $x$ belongs to $B$ is determined at a countable ordinal in the jump hierarchy relative to $x$ and the Borel code $b$ for $B$. Let $C$ be the set of triples $(x, b, s)$ such that $s$ is the Skolem function verifying the relevant hyperarithmetic statement about $x$ and $b$.

COROllary 2.6. Every uncountable Borel subset of ${ }^{\omega} \omega$ is a continuous injective image of the sum of ${ }^{\omega} \omega$ with a countably infinite discrete set.

Proof. By Theorem 2.5, it is enough to show that every uncountable closed set is a continuous injective image of the sum of ${ }^{\omega} \omega$ with a countably infinite discrete set. This follows from the Cantor-Bendixson analysis of closed sets.

Now, we prove the converse.

TheOREm 2.7 (Luzin-Suslin). Suppose that $B$ is a Borel subset of ${ }^{\omega} \omega$, and that $f$ is a continuous function that is injective on $B$. Then the range of $f$ applied to $B$ is a Borel set.

Proof. Clearly, $f^{\prime \prime} B$ is a $\Sigma_{1}^{1}$ set. Let $b$ be a real parameter used in the Borel definition of $B$

Note, if $x \in f^{\prime \prime} B$ then

$$
f^{-1}(x)(n)=m \Longleftrightarrow(\exists z)[z \in B \text { and } z(n)=m \text { and } f(z)=x]
$$

So, $f^{-1}(x)$ is uniformly $\Sigma_{1}^{1}(x, f, b)$ definable and similarly $\Delta_{1}^{1}(x, f, b)$ definable. Consequently, $x \in f^{\prime \prime} B$ if and only if there is an ordinal $\beta$ less than $\omega_{1}^{x, f, b}$ and a $z$ in $L_{\beta}[x, f, b]$ such that $L_{\beta}[x, f, b]$ satisfies the conditions that $z \in B$ and $f(z)=x$. This gives a $\Pi_{1}^{1}$ definition of $f^{\prime \prime} B$.

Consequently, $f^{\prime \prime} B$ is a $\boldsymbol{\Pi}_{1}^{1}$ set, and therefore is a Borel set.

COROLlary 2.8. The Borel sets are exactly the injective continuous images of the sum of ${ }^{\omega} \omega$ with a countably infinite discrete set.

2.3. Sierpiński's Problem. Sierpiński (1936) raised the question whether there is an analogous version of Theorem 2.7 for the analytic sets.

Question 2.9 (Sierpiński (1936)). Does there exist a subset $U$ of ${ }^{\omega} \omega$ such that for every uncountable $\boldsymbol{\Sigma}_{1}^{1}$ set $A$, there is a continuous function $f$ that maps $U$ bijectively to $A$ ?

Of course, the complete $\boldsymbol{\Sigma}_{1}^{1}$ set has all of the desired properties except for the key property that $f$ should be injective. 


\subsubsection{Solution to Sierpiński's problem.}

THEOREM 2.10 (Slaman (1999)).

- There is no $\boldsymbol{\Sigma}_{1}^{1}$ set which satisfies all of the Sierpinski properties.

- There is a set U which satisfies all of the Sierpiński properties.

Proof. The proof of the first claim is short enough to include here, but we will only point to (Slaman, 1999) or (Hjorth, n.d.) for the proof of the second claim.

To prove the first claim, we proceed by contradiction. Let $U$ be an $\boldsymbol{\Sigma}_{1}^{1}$ set such that for every uncountable $\boldsymbol{\Sigma}_{1}^{1}$ set $A$, there is a continuous function $f$ that maps $U$ bijectively to $A$.

Let $f$ be a continuous bijection from $U$ to ${ }^{\omega} \omega$. As above, for $x \in{ }^{\omega} \omega, f^{-1}(x)$ is $\Delta_{1}^{1}(x, f)$. Then for all $y \in{ }^{\omega} \omega, y \in U$ if and only if $y=f^{-1}(f(y))$, and so $U$ is $\boldsymbol{\Delta}_{1}^{1}$.

Now apply the Luzin-Suslin theorem. Since the complete $\Sigma_{1}^{1}$ set of reals is a continuous bijective image of $U$, it is $\boldsymbol{\Delta}_{1}^{1}$, an impossibility.

2.3.2. Hjorth's theorem. Slaman (1999) raised the question, is there a projective $U$ as in Theorem 2.10? Hjorth (n.d.) gave the best possible example.

THEOREM 2.11 (Hjorth (n.d.)). There is a $\mathbf{\Pi}_{1}^{1}$ set $U$ which provides a positive solution to Sierpinski's problem. In fact, the set of reals which are hyperjumps

$$
\mathcal{H}=\left\{\mathcal{O}^{x}: x \in{ }^{\omega} \omega\right\}
$$

is such a set $U$.

It takes us beyond the usual recursion theoretic horizon, but Sierpiński's question is sensible at any level of the projective hierarchy.

THEOREM 2.12 (Hjorth). The following statements are equivalent.

1. Every uncountable $\boldsymbol{\Pi}_{1}^{1}$ set contains a perfect set. Equivalently, for all $z \in{ }^{\omega} \omega$, $\aleph_{1}$ is inaccessible in $L[z]$.

2. Every uncountable $\boldsymbol{\Sigma}_{2}^{1}$ set is the continuous injective image of $\mathcal{H}$.

Though not contained in its entirety, much of the proof of Theorem 2.12 contained in (Hjorth, n.d.).

2.3.3. Harrington's theorem. In Theorem 2.10, we observed that no analytic set could be a universal injective preimage for all of the uncountable analytic sets. We argued that if $U$ is analytic and ${ }^{\omega} \omega$ is a continuous injective image of $U$, then $U$ is Borel. Then, we concluded that $U$ could not be universal.

Steel raised the question, could an analytic set be a universal injective preimage for all of the properly analytic sets? Harrington provided the answer.

THEOREM 2.13 (Harrington). The following statements are equivalent.

1. There is a $\boldsymbol{\Sigma}_{1}^{1}$ set $A$ such that every non-Borel analytic set is a continuous injective image of $A$.

2. For every real $x$, the real $x^{\#}$ exists. Equivalently, $\boldsymbol{\Sigma}_{1}^{1}$-determinacy holds.

In fact, under $\boldsymbol{\Sigma}_{1}^{1}$-determinacy, every non-Borel $\boldsymbol{\Sigma}_{1}^{1}$ set satisfies the property of $A$ in (1).

It may seem that we have wandered far from our recursion theoretic home base, but that is not the case. The principal ingredients in Harrington's proof are the Kleene Fixed Point Theorem and Steel forcing over $\Sigma_{1}$-admissible sets. What could be more recursion theoretic? 


\section{Directions for further investigation}

In the previous section, we saw that a recursion theorist can travel into the set theoretic domain without becoming completely lost. Now, we turn to some open questions which can be found there.

\subsection{Harrington's question.}

Definition 3.1. For subsets $A$ and $B$ of ${ }^{\omega} \omega$, we say $A \geq_{W} B$ if there is a continuous function $f$ such that for all $x$ in ${ }^{\omega} \omega, x \in B$ if and only if $f(x) \in A$.

When $A \geq_{W} B$, we say that $B$ is Wadge reducible to $B$. Wadge reducibility between sets of reals is analogous to many-one reducibility between sets of natural numbers.

Theorem 3.2 (Wadge). Under the Axiom of Determinacy, for all subsets $A$ and $B$ of ${ }^{\omega} \omega$, either $A \geq_{W} B$ or $\bar{B} \geq_{W} A$, where $\bar{B}$ is the complement of $B$ in ${ }^{\omega} \omega$.

Corollary 3.3. For any two properly $\Sigma_{1}^{1}$ sets $A$ and $B, A \equiv_{W} B$.

Steel (1980), and later Harrington in more generality, sharpened Corollary 3.3 to add injectivity to the reducing function.

TheOREM 3.4 (Steel (1980)). Under the Axiom of Determinacy, for any two properly $\boldsymbol{\Sigma}_{1}^{1}$ sets $A$ and $B$ there is an injective continuous function $f$ such that for all $x, x \in B$ if and only if $f(x) \in A$.

Corollary 3.5. Under the Axiom of Determinacy, for any two properly $\boldsymbol{\Sigma}_{1}^{1}$ subsets $A$ and $B$ of ${ }^{\omega} \omega$, there is a Borel permutation $\pi$ of ${ }^{\omega} \omega$ such that $\pi[A]=B$.

TheOREM 3.6 (Harrington (1978)). Suppose that every $\boldsymbol{\Sigma}_{1}^{1}$ subset $A$ of ${ }^{\omega} \omega$ is either Borel or $\geq_{W}$-complete. Then the Axiom of Determinacy holds for $\boldsymbol{\Sigma}_{1}^{1}$ sets.

Harrington's proof of Theorem 3.6 involves a fair amount of set theory. Though off topic for us, Harrington has raised the interesting question of whether Theorem 3.6 is provable from the usual axioms of second order arithmetic. More on topic is his question of whether determinacy follows from the weaker hypothesis that the Wadge degrees of the $\boldsymbol{\Sigma}_{1}^{1}$ sets are linearly ordered.

Question 3.7 (Harrington). Suppose that any two $\boldsymbol{\Sigma}_{1}^{1}$ non-Borel subsets of $\omega_{\omega}$ are $\geq_{W}$ comparable. Does the Axiom of Determinacy hold for $\boldsymbol{\Sigma}_{1}^{1}$ sets?

3.2. Hjorth's question. Let $D$ denote a countable discrete set.

Question 3.8 (Hjorth (n.d.)). Suppose that for any uncountable $\boldsymbol{\Sigma}_{2}^{1}$ set $B$ and $\boldsymbol{\Pi}_{1}^{1}$ non-Borel set $C, B$ is the continuous image of $C \oplus D$. Then, must $\boldsymbol{\Sigma}_{1}^{1}$ determinacy hold?

\subsection{Comparing the Wadge and Sierpiński orderings.}

Definition 3.9. For subsets $A$ and $B$ of $\omega_{\omega} \omega$, say that $A \geq_{S} B$ if there is a function $f$ which is partial recursive in some real parameter whose restriction to $A$ is a bijection from $A$ to $B$.

Question 3.10. 1. What is the structure of $\geq_{S}$ ?

2. Is there a relationship between $\geq_{W}$ and $\geq_{S}$ ?

We first heard the following question from Hjorth, who also told us that it is not original to him.

QUESTION 3.11. What is the structure of $\geq_{S}$ on countable metric spaces? 


\subsection{Steel's question.}

Definition 3.12. Say that two subsets $A$ and $B$ are homeomorphically equivalent if there is a homeomorphism $f:{ }^{\omega} \omega \rightarrow{ }^{\omega} \omega$ such that $f[A]=B$.

Question 3.13 (Steel). Is there a natural classification of the analytic sets up to homeomorphic equivalence?

\section{References}

Harrington, L. A. (1978). Analytic determinacy and 0\#, J. Symbolic Logic 20: 685-693.

Hjorth, G. (n.d.). Continuous images of coanalytic sets, Logic Colloquium'98 Prague. to appear. Kechris, A. S. (1995). Classical Descriptive Set Theory, Vol. 156 of Graduate Texts in Mathematics, Springer-Verlag, Heidelberg.

Sacks, G. E. (1990). Higher Recursion Theory, Perspectives in Mathematical Logic, SpringerVerlag, Heidelberg.

Sierpiński, W. (1936). Problem 70, Fund. Math. 26: 334.

Slaman, T. A. (1999). On a question of Sierpiński, Fund. Math. 159(2): 153-159.

Steel, J. R. (1980). Analytic sets and Borel isomorphisms, Fund. Math. 108(2): 83-88.

University of California, Berkeley, Berkeley, CA 94720-3840, USA

E-mail address: slaman@math.berkeley.edu 